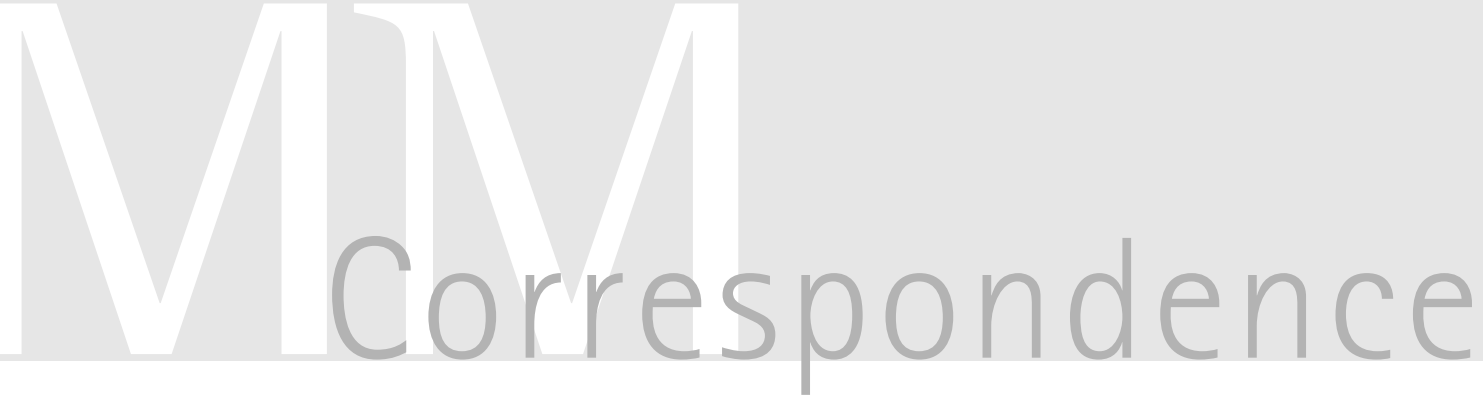

\title{
Microbiological, histopathological and clinical changes in chronic infected wounds after citric acid treatment
}

Citric acid treatment of chronic infected wounds offers excellent results. It has been found to be effective against a variety of bacteria causing wound infections. In vitro studies have revealed the efficacy of citric acid against bacteria resistant to multiple antibiotics. Clinical results with this treatment showed early formation of healthy granulation tissue and enhancement of the healing process (Nagoba et al., 1998, 1999, 2002).

In the present study, an attempt has been made to study the microbiological and histopathological response of chronic infected wounds following citric acid application (locally), and its correlation with clinical manifestations, in two cases.
The citric acid was prepared in distilled water: $100 \mathrm{ml}$ distilled water in a screw cap glass bottle was sterilized by autoclaving at $121{ }^{\circ} \mathrm{C}$ at $1.05 \mathrm{~kg} \mathrm{~cm}^{-2}$ pressure for $15 \mathrm{~min}$ to which $3 \mathrm{~g}$ citric acid was added taking all sterile precautions.

A 65-year-old male, non-diabetic patient presented with a non-healing ulcer over the dorsum of his left foot, which was resistant to the traditional therapy that he had received for 1 month. The ulcer extended over whole of the dorsum, the edges were scarred with evident unhealthy granulation tissue and slough. The underlying tendons were exposed (Fig. 1a). The ulcer was evaluated for microbiological, histopathological and (a)

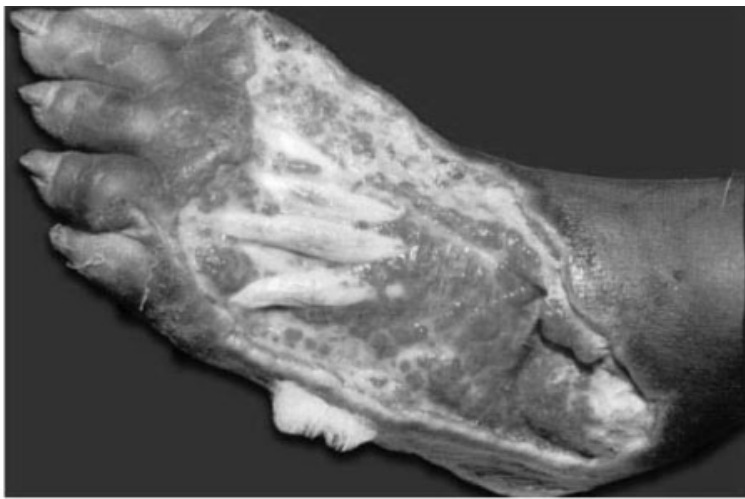

(b)

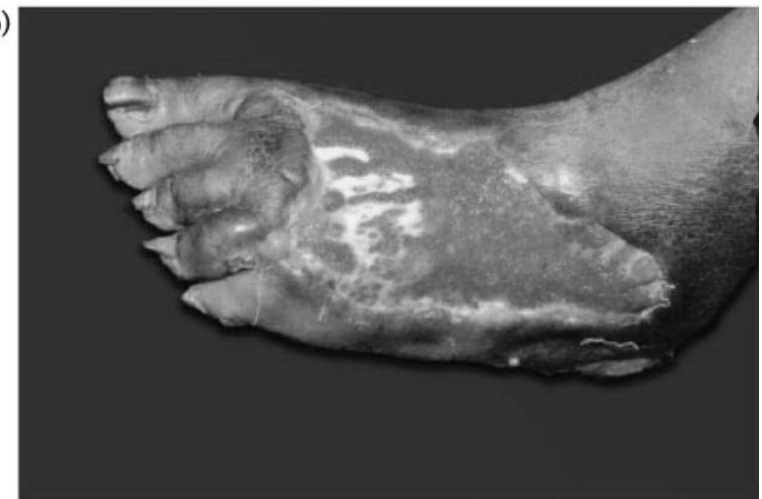

Fig. 1. Non-healing ulcer before application of citric acid (a) and after six applications of citric acid (b).

clinical changes. The evaluation was carried out in two phases, i.e. pre-citric acid application and post-citric acid application. The culture of the exudates prior to application of citric acid yielded Staphylococcus aureus resistant to ampicillin, cotrimoxazole, erythromycin, gentamicin, cephalexin, ceftazidime and pefloxacin, and susceptible to cloxacillin, chloramphenicol and ciprofloxacin. The MIC of citric acid by the broth dilution method was found to be $900 \mu \mathrm{g} \mathrm{ml}^{-1}$ for S. aureus. In the pre-citric acid application, histopathological study revealed areas of necrosis and dense inflammatory exudates composed of polymorphonuclear leukocytes (PML), macrophages and lymphocytes.

As a treatment strategy, $3 \%$ citric acid was applied locally in the form of wound irrigation and wound wash, and by the placing of a pad soaked with citric acid on the wound once daily. In this way six applications were carried out before repeating the biopsy and swab for culture. The patient did not receive any antibiotics during the course of the study.

Post-citric acid application, the repeat culture of a swab had no growth and histopathological findings had an encouraging revelation of blood vessels and proliferating fibroblasts. Inflammatory exudates showed sparse PML and clinically healthy granulation tissue was evident (Fig. 1b).

An 80-year-old non-diabetic male presented with a non-healing ulcer of 2 months duration over the lateral aspect of his left ankle joint. The clinical appearance of the ulcer had a very bad look with abundant slough and active pus discharge. A culture of the exudates yielded $S$. aureus and Escherichia coli. The S. aureus was resistant to ampicillin, cotrimoxazole, chloramphenicol, erythromycin, gentamicin, pefloxacin, cephalexin and ceftazidime, and susceptible to cloxacillin and ciprofloxacin. The E. coli was 
susceptible to chloramphenicol, cephalexin, ciprofloxacin and pefloxacin. The MIC of citric acid by broth dilution method was found to be $900 \mu \mathrm{g} \mathrm{ml}^{-1}$ for S. aureus and $1500 \mu \mathrm{g} \mathrm{ml}^{-1}$ for E. coli. The comparative pre- and post-application histopathology study revealed the same results as in the first case described above. The reculture of a swab from the ulcer after two citric acid applications only had six colonies of $S$. aureus, with complete elimination of E. coli. Clinically, the granulation tissue covering the ulcer was satisfactory.

The effectiveness of citric acid in the management of chronic wound infections has been reported and acclaimed. Citric acid has been found highly effective against almost all bacterial pathogens commonly involved in wound infections, including notorious pathogens like Pseudomonas aeruginosa (citric acid MIC in the range $800-1000 \mu \mathrm{g} \mathrm{ml}^{-1}$ ), which is a nuisance pathogen in many of these cases and certainly delays healing (Nagoba et al., 1998, 1999, 2002). In the present study microbiological examination showed that citric acid caused a significant reduction in the number of infectious agents and histopathological examination showed that citric acid boosts fibroblastic growth and neovascularization, which in turn increases microcirculation of wound that enables the formation of healthy granulation tissue thereby leading to faster healing of the wound.

The results show that microbiological and histopathological findings correlate fairly well with clinical findings. Hence it is concluded that citric acid therapy can be a good alternative for traditional therapies, while dealing with chronic infected wounds. Compared with the questionable efficacy and toxicity of presently available local antiseptic preparations (Lineaweaver et al., 1985; Eaglstein \& Falanga, 1997; Russell et al., 2004) citric acid, being a natural product, is non-toxic, has no side effects and has potential to control infections, and may be preferred as a good wound dressing agent.

\section{B. S. Nagoba, ${ }^{1}$ R. C. Gandhi, ${ }^{2}$ B. J. Wadher, ${ }^{3}$ R. M. Potekar ${ }^{4}$ and S. M. Kolhe ${ }^{5}$}

${ }^{1}$ Department of Microbiology, Maharashtra Institute of Medical Sciences and Research, Medical College and Hospital, Latur 413 531, Maharashtra, India

${ }^{2}$ Department of Surgery, Maharashtra Institute of Medical Sciences and Research, Medical College and Hospital, Latur 413 531, Maharashtra, India

${ }^{3}$ Department of Microbiology, Medical Microbiology Research Laboratory, Napur University, Nagpur, India
${ }^{4}$ Department of Pathology, BLDE's B. M. Patil Medical College, Bijapur, India

${ }^{5}$ Department of Pharmacology, Maharashtra Institute of Medical Sciences and Research Medical College, Latur, India

Correspondence: B. S. Nagoba (dr_bsnagoba@yahoo.com or bsnagoba@indiatimes.com)

Eaglstein, W. H. \& Falanga, V. (1997). Chronic wounds. Surg Clin North Am 77, 689-700.

Lineaweaver, W., Howard, R., Soucy, D., McMorris, S., Freeman, J., Crain, C., Robertson, J. \& Rumley, T. (1985). Topical antimicrobial toxicity. Arch Surg 120, 267-270.

Nagoba, B. S., Deshmukh, S. R., Wadher, B. J., Mahabaleshwar, L., Gandhi, R. C., Kulkarni, P. B., Mane, V. A. \& Deshmukh, J. S. (1998). Treatment of superficial pseudomonal infections with citric acid: an effective and economical approach. J Hosp Infect 40, 155-157.

Nagoba, B. S., Pathan, A. B. \& Wadher, B. J. (1999). Citric acid treatment of superficial nosocomial infections: a new era in the antimicrobial chemotherapy. Kuwait Med Journal 31, 72-74.

Nagoba, B. S., Wadher, B. J. \& Chandorkar, A. G. (2002). Citric acid treatment of non-healing ulcers in leprosy patients. Br J Dermatol 146, 1101.

Russell, R. C. G., Williams, N. S. \& Bulstrode, C. J. K. (2004). Bailey \& Love's Short Practice of Surgery, 24th edn. London: Arnold. 